\title{
Products and Covering of Lattice-valued Finite Automata
}

\author{
Lou Quanfu, Hu Zhonggang \\ Nanchang Institute of Science \& Technology, Nanchang, Jiangxi, 330108, China \\ Email:zhonggang-hu@163.com
}

Keywords: lattice-valued finite automata; homomorphism; full direct product; restricted product; cascade product; wreath product; coverings.

\begin{abstract}
In this paper, the new concept of covering for lattice-valued finite automata was given. The product relations between various lattice-valued finite automata, the covering relations between various products of two lattice-valued finite automata, and covering relations between products of two lattice-valued finite automata and products of the other two lattice-valued finite automata which cover them are studied.
\end{abstract}

\section{Introduction}

Since the fuzzy set theory was put forward in [1], the research on automata theory and code theory has also unfolded in [2] with the method of fuzzy set. After that, the research and application of fuzzy automata became more and more in-depth. [3] began to study the algebra of fuzzy automata. [4] has made a detailed study of the algebraic properties of fuzzy automata. In 2003, Li Yongming in [5] proposed the theory of Lattice-valued automata and its language, and built the words-based computational model on the theory of more extensive Lattice-valued automata. In automata theory, product is one of the basic operations, and the product and covering relation of different forms play a very important role in the decomposition of automata. The products of lattice-valued finite automata are studied in [6]. [3] studies the product and covering relation of a fuzzy finite state machine. A new covering concept is given in this paper, and the covering relation between the products of the Lattice-valued finite automata given in [6] is studied. The covering relation between the product of lattice-valued finite state machines and the product of those covering them is also discussed.

\section{Basic Concepts and Symbols}

Definition 2.1 ${ }^{[7]}$ A lattice-valued finite automata (LFA) is a five tuple, $M=(Q, X, Y, \mu, \sigma)$,in which $Q, X, Y$ are non-empty finite state set, non-empty finite input symbol set, non-empty finite output symbol set respectively. $\mu$ is a L-fuzzy subset in $Q \times X \times Q$, that is $\mu: Q \times X \times Q \rightarrow L$, which is called fuzzy state transfer function. $\sigma$ is a L-fuzzy subset in $Q \times X \times Y$, that is, $\sigma: Q \times X \times Y \rightarrow L$, which is called fuzzy output function. For a lattice-valued finite automata, the following conditions are satisfied:

$$
(\forall q \in Q)(\forall x \in X)((\exists p \in Q), \mu(q, x, p)>0 \Leftrightarrow(\exists y \in Y), \sigma(q, x, y)>0)
$$

Because only the transfer structure of automata is discussed, the lattice-valued finite automata in the definition above is expressed as $M=(Q, X, \mu)$, which is called a finite state machine. We agree that semi-group operations " $\bullet "$ here are commutative.

Definition 2.2 $2^{8]}$ Suppose $M=(Q, X, \mu)$ is a lattice-valued finite state machine, among which $\forall x \in X^{*}, a \in X . \Lambda$ is an empty character, $X^{*}$ represents the set of all strings of finite length on the set $X . \mu^{*}: Q \times X^{*} \times Q \rightarrow L$ is defined as follows:

$$
\mu^{*}(q, \Lambda, p)=\left\{\begin{array}{l}
1, q=p \\
0, q \neq p
\end{array}, \quad \mu^{*}(q, x a, p)=\underset{r \in Q}{\vee}\left\{\mu^{*}(q, x, r) \bullet \mu(r, a, p)\right\}\right.
$$




\section{The Covering of Lattice-valued Finite State Machines}

Definition 3.1 Suppose $M_{i}=\left(Q_{i}, X_{i}, \mu_{i}\right)$ is a lattice-valued finite state machine, $i=1,2 \cdot \eta: Q_{2} \rightarrow Q_{1}$ is a partial function,and $\xi: X_{1} \rightarrow X_{2}$ is another function.Expand $\xi$ to $\xi^{*}$, making $\xi^{*}(\Lambda)=\Lambda, \xi^{*}(x)=\xi\left(x_{1}\right) \xi\left(x_{2}\right) \cdots$

$\xi\left(x_{n}\right)$, among which $x=x_{1} x_{2} \cdots x_{n}, x_{i} \in X_{1}^{*}, i=1,2, \cdots, n$. Then $(\eta, \xi)$ is called a covering of $M_{2} \rightarrow M_{1}$,

marked as $M_{2} \leq M_{1}$. If satisfied: $\mu_{1}\left(\eta\left(q_{2}\right), x, \eta\left(p_{2}\right)\right) \leq \mu_{2}\left(q_{2}, \xi(x), p_{2}\right), \forall p_{2}, q_{2} \in Q_{2}, x \in X_{1}$

Proposition 3.1 Suppose $M_{i}=\left(Q_{i}, X_{i}, \mu_{i}\right)$ is a lattice-valued finite state machine, $i=1,2 .(\eta, \xi)$ is a covering of $M_{2} \rightarrow M_{1}$, then $\mu_{1}^{*}\left(\eta\left(q_{2}\right), x, \eta\left(p_{2}\right)\right) \leq \mu_{2}^{*}\left(q_{2}, \xi(x), p_{2}\right), \forall p_{2}, q_{2} \in Q_{2}, x \in X_{1}^{*}$.

Proof The induction of character length $n$. When $n=2$, making $x=x_{1} x_{2}, y=y_{1} y_{2}$,

$$
\begin{aligned}
& \mu_{1}^{*}\left(\eta\left(q_{2}\right), x y, \eta\left(p_{2}\right)\right)=\underset{r \in Q}{\vee}\left\{\mu_{1}\left(\eta\left(q_{2}\right), x_{1}, r\right) \wedge \mu_{1}\left(r, x_{2}, \eta\left(p_{2}\right)\right)\right\} \\
& \leq \underset{r \in Q_{1}}{\vee}\left\{\mu_{2}\left(q_{2}, x_{1}, \eta\left(p_{2}\right)\right) \wedge \mu_{2}\left(r, x_{2}, p_{2}\right) \mid \eta\left(r_{1}\right)=r\right\} \leq \mu_{2}^{*}\left(q_{2}, \xi\left(x_{1} x_{2}\right), p_{2}\right)
\end{aligned}
$$

Suppose the conclusion is set up when $n=k$, then $n=k+1$, making $x=\prod_{i=1}^{k+1} x_{i}, x_{i} \in X_{1}$.

$$
\begin{aligned}
& \mu_{1}^{*}\left(\eta\left(q_{2}\right), \prod_{t=1}^{k+1} x_{i}, \eta\left(p_{2}\right)\right)=\vee\left\{\mu_{1}^{*}\left(\eta\left(q_{2}\right), \prod_{i=1}^{k} x_{i}, r_{1}\right) \wedge \mu_{1}\left(r_{1}, x_{k+1}, \eta\left(p_{2}\right)\right) \mid r_{1} \in Q_{1}\right\} \\
& =\vee\left\{\mu_{1}^{*}\left(\eta\left(q_{2}\right), \prod_{t=1}^{k} x_{i}, \eta\left(r_{2}\right)\right) \wedge \mu_{1}\left(\eta\left(r_{2}\right), x_{k+1}, \eta\left(p_{2}\right)\right) \mid \eta\left(r_{2}\right)=r_{1}, r_{1} \in Q_{1}\right\} \\
& \leq \vee\left\{\mu_{2}^{*}\left(q_{2}, \xi\left(\prod_{i=1}^{k} x_{i}\right), \eta\left(r_{2}\right)\right) \wedge \mu_{1}\left(\eta\left(r_{2}\right), x_{k+1}, \eta\left(p_{2}\right)\right) \mid r_{2} \in Q_{2}\right\} \\
& \leq \vee\left\{\mu_{2}^{*}\left(q_{2}, \xi\left(\prod_{i=1}^{k} x_{i}\right), r_{2}\right) \wedge \mu_{2}\left(r_{2}, \xi\left(x_{k+1}\right), \eta\left(p_{2}\right)\right) \mid r_{2} \in Q_{2}\right\}=\mu_{2}^{*}\left(q_{2}, \xi\left(\prod_{i=1}^{k+1} x_{i}\right), p_{2}\right) .
\end{aligned}
$$

Definition 3.2 ${ }^{[8]}$ Suppose $M_{1}=\left(Q_{1}, X_{1}, \mu_{1}\right)$ and $M_{2}=\left(Q_{2}, X_{2}, \mu_{2}\right)$ are two lattice-valued finite state machines. (1)A pair of mappings $(\alpha, \beta), \alpha: Q_{1} \rightarrow Q_{2}, \beta: X_{1} \rightarrow X_{2}$ are homomorphic,marked as $(\alpha, \beta): M_{1} \rightarrow M_{2}$, suppose $\mu_{1}(q, x, p) \leq \mu_{2}(\alpha(q), \beta(x), \alpha(p)), \forall p, q \in Q_{1}, \forall x \in X_{1}$. (2) $(\alpha, \beta)$ is called a strong homomor-phic mapping,suppose

$$
\mu_{2}(\alpha(q), \beta(x), \alpha(p))=\vee\left\{\mu_{1}(q, x, t) \mid t \in Q_{1}, \alpha(t)=\alpha(p)\right\}, \forall p, q \in Q_{1}, \forall x \in X_{1} .
$$

If $\alpha, \beta$ is surjective (injective), then homomorphism $(\alpha, \beta): M_{1} \rightarrow M_{2}$ is surjective (injective); If $\alpha, \beta$ is one-to-one mapping, then homomorphism (strong homomorphism) $(\alpha, \beta): M_{1} \rightarrow M_{2}$ is called isomorph-ism (strong isomorphism).

Note: If $X_{1}=X_{2}, \beta$ is an identity mapping, then simply marking $\alpha: M_{1} \rightarrow M_{2}$, then correspondently naming $\alpha$ as homomorphism (strong homomorphism).

Lemma 3.1 ${ }^{[8]}$ Suppose $M_{i}=\left(Q_{i}, X_{i}, \mu_{i}\right)$ is a lattice-valued finite state machine, $i=1,2$.If $(\alpha, \beta): M_{1} \rightarrow M_{2}$ is a strong homomorphism, and $\alpha$ is an injective mapping, then

$$
\forall p, q \in Q_{1}, \forall x \in X_{1} \text {, making } \mu_{2}(\alpha(q), \beta(x), \alpha(p))=\mu_{1}(q, x, p) \text {. }
$$

Theorem 3.1 Suppose $M_{i}=\left(Q_{i}, X_{i}, \mu_{i}\right)$ is a lattice-valued finite state machine, $i=1,2$.If $(\alpha, \beta): M_{1} \rightarrow M_{2}$ is a homomorphism,then(1)If this homomorphism is an epimorphism, and $\alpha$ is an injective mapping, then $M_{2} \leq M_{1}$;(2)If $\alpha$ is an injective mapping, then $M_{1} \leq M_{2}$.

Proof (1) $(\alpha, \beta): M_{1} \rightarrow M_{2}$ is a strong homomorphism, so there exist full functions $\alpha: Q_{1} \rightarrow Q_{2}$ and $\beta: X_{1} \rightarrow X_{2}$, making $\eta=\alpha: Q_{1} \rightarrow Q_{2}, \xi: X_{2} \rightarrow X_{1} ;$ As $\beta$ is a full function, 
then $\forall x_{2} \in X_{2} \quad$,at least existing $x_{1} \in X_{1}$, which makes $\beta\left(x_{1}\right)=x_{2}$, thus $\xi\left(x_{2}\right)=a$.Furthermore, $(\alpha, \beta)$ is a strong homomorphism, and $\alpha$ is an injective mapping. Based on Lemma 3.1, which makes $\forall p, q \in Q_{1}, \forall x \in X_{1}$, thus $\mu_{2}(\alpha(q), \beta(x), \alpha(p))=\mu_{1}(q, x, p)$.If $\xi\left(x_{2}\right)=x_{1} \quad$ we can conclude that $\mu_{2}\left(\alpha(q), x_{2}, \alpha(p)\right)=\mu_{2}\left(\alpha(q), \beta\left(x_{1}\right), \alpha(p)\right)=\mu_{1}\left(q, x_{1}, p\right)=\mu_{1}\left(q, \xi\left(x_{1}\right), p\right)$, for $(\eta, \xi)$ is a covering of $M_{1} \rightarrow M_{2}$, thus $M_{2} \leq M_{1}$.

(2) $(\alpha, \beta): M_{1} \rightarrow M_{2}$ is a homomorphism, so there exist mappings $\alpha: Q_{1} \rightarrow Q_{2}$ and $\beta: X_{1} \rightarrow X_{2}$, making $\forall p_{1}, q_{1} \in Q_{1}, \forall x_{1} \in X_{1} \quad$,then $\quad \mu_{1}\left(q_{1}, x_{1}, p_{1}\right) \leq \mu_{2}\left(\alpha\left(q_{1}\right), \beta\left(x_{1}\right), \alpha\left(p_{1}\right)\right) \quad$,making $\eta: Q_{2} \rightarrow Q_{1}, \eta\left(q_{2}\right)$,If $\alpha\left(q_{1}\right)=q_{2}$, thus $q_{1}$ is uniquely determined because $\alpha$ is an injective mapping, thus $\eta$ is a part of full function, making $\xi=\beta: X_{1} \rightarrow X_{2}$, thus $\forall p_{2}, q_{2} \in Q_{2}, \forall x_{1} \in X_{1}$, Then $\mu_{1}\left(\eta\left(q_{2}\right), x_{1}, \eta\left(p_{2}\right)\right) \leq \mu_{2}\left(q_{2}, \beta\left(x_{1}\right), p_{2}\right)$, Therfore, $(\eta, \xi)$ is a covering of $M_{2} \rightarrow M_{1}$, that is, $M_{1} \leq M_{2}$.

Theorem 3.2 Suppose $M_{i}=\left(Q_{i}, X_{i}, \mu_{i}\right)$ is a lattice-valued finite state machine, $i=1,2$. If $(\alpha, \beta): M_{1} \rightarrow M_{2}$ is a homomorphism, then(1)If this homomorphism is an epimorphism, and $\alpha$ is an injective mapping, then $M_{2} \leq M_{1}$;(2)If $\alpha$ is an injective mapping, then $M_{1} \leq M_{2}$.

\section{Products of Lattice-valued Finite Automata}

Definition 4.1 ${ }^{[7]}$ Suppose $M_{i}=\left(Q_{i}, X_{i}, \mu_{i}\right)$ is a lattice-valued finite state machine, $i=1,2$, naming $M_{1} \times M_{2}=\left(Q_{1} \times Q_{2}, X_{1} \times X_{2}, \mu_{1} \times \mu_{2}\right)$ as a full direct product of $M_{1}$ and $M_{2}$, among which $\mu_{M_{1} \times M_{2}}\left(\left(Q_{1} \times Q_{2}\right) \times\left(X_{1} \times X_{2}\right) \times\left(Q_{1} \times Q_{2}\right)\right) \rightarrow L, \forall\left(q_{1}, q_{2}\right),\left(p_{1}, p_{2}\right) \in Q_{1} \times Q_{2}, \forall\left(x_{1}, x_{2}\right) \in X_{1} \times X_{2}$, making $\mu_{M_{1} \times M_{2}}\left(\left(q_{1}, q_{2}\right),\left(x_{1}, x_{2}\right),\left(p_{1}, p_{2}\right)\right)=\mu_{M_{1}}\left(q_{1}, x_{1}, p_{1}\right) \wedge \mu_{M_{2}}\left(q_{2}, x_{2}, p_{2}\right)$.

Definition 4.2 ${ }^{[7]}$ Suppose $M_{i}=\left(Q_{i}, X, \mu_{i}\right)$ is a lattice-valued finite state machine, $i=1,2$, naming $M_{1} \wedge M_{2}=\left(Q_{1} \times Q_{2}, X, \mu_{1} \wedge \mu_{2}\right)$ as a retricted direct product of $M_{1}$ and $M_{2}$, among which

$$
\begin{gathered}
\mu_{M_{1} \wedge M_{2}}\left(\left(Q_{1} \times Q_{2}\right) \times X \times\left(Q_{1} \times Q_{2}\right)\right) \rightarrow L, \forall\left(q_{1}, q_{2}\right),\left(p_{1}, p_{2}\right) \in Q_{1} \times Q_{2}, x \in X \text {, making } \\
\mu_{M_{1} \wedge M_{2}}\left(\left(q_{1}, q_{2}\right), x,\left(p_{1}, p_{2}\right)\right)=\mu_{M_{1}}\left(q_{1}, x, p_{1}\right) \wedge \mu_{M_{2}}\left(q_{2}, x, p_{2}\right) .
\end{gathered}
$$

Definition 4.3 $3^{[7]}$ Suppose $M_{i}=\left(Q_{i}, X_{i}, \mu_{i}\right)$ is a lattice-valued finite state machine, $i=1,2$, naming $M_{1} \circ M_{2}=\left(Q_{1} \times Q_{2}, X_{1}^{Q_{2}} \times X_{2}, \mu_{1} \circ \mu_{2}\right)$ as a wreath product of $M_{1}$ and $M_{2}$, among which $\mu_{M_{1} \circ M_{2}}\left(\left(Q_{1} \times Q_{2}\right)\right.$

$\left.\times\left(X_{1}^{Q_{2}} \times X_{2}\right) \times\left(Q_{1} \times Q_{2}\right)\right) \rightarrow L, X_{1}^{Q_{2}}=\left\{f \mid f: Q_{2} \rightarrow X_{1}\right\}, \forall\left(\left(q_{1}, q_{2}\right),\left(f, x_{2}\right),\left(p_{1} \times p_{2}\right)\right) \in\left(Q_{1} \times Q_{2}\right) \times\left(X_{1}^{Q_{2}} \times\right.$ $\left.X_{2}\right) \times\left(Q_{1} \times Q_{2}\right)$, making $\mu_{M_{1} \circ M_{2}}\left(\left(q_{1}, q_{2}\right),\left(f, x_{2}\right),\left(p_{1}, p_{2}\right)\right)=\mu_{M_{1}}\left(q_{1}, f\left(q_{2}\right), p_{1}\right) \wedge \mu_{M_{2}}\left(q_{2}, x_{2}, p_{2}\right)$.

Definition $4.4^{[7]}$ Suppose $M_{i}=\left(Q_{i}, X_{i}, \mu_{i}\right)$ a lattice-valued finite state machine, $i=1,2$, naming $M_{1} \omega M_{2}=\left(Q_{1} \times Q_{2}, X_{2}, \mu_{1} \omega \mu_{2}\right)$ as a cascade product of $M_{1}$ and $M_{2}$, among which

$$
\mu_{M_{1} \omega M_{2}}\left(\left(Q_{1} \times Q_{2}\right) \times X_{2} \times\left(Q_{1} \times Q_{2}\right)\right) \rightarrow L, \omega: Q_{2} \times X_{2} \rightarrow X_{1} \text { is a function, } \forall\left(q_{1}, q_{2}\right),\left(p_{1}, p_{2}\right) \in Q_{1} \times Q_{2}
$$
, $\forall x_{2} \in X_{2}$,making $\mu_{M_{1} \omega M_{2}}\left(\left(q_{1}, q_{2}\right), x_{2},\left(p_{1}, p_{2}\right)\right)=\mu_{M_{1}}\left(q_{1}, \omega\left(q_{2}, x_{2}\right), p_{1}\right) \wedge \mu_{M_{2}}\left(q_{2}, x_{2}, p_{2}\right)$.

Theorem 4.1 Suppose $M_{i}=\left(Q_{i}, X_{i}, \mu_{i}\right)$ is a lattice-valued finite state machine, $i=1,2$.then

$$
M_{1} \wedge M_{2} \leq M_{1} \times M_{2}
$$

$$
\text { , among }
$$

which

$X_{1}=X_{2}=X$, (2) $M_{1} \omega M_{2} \leq M_{1} \circ M_{2}$, (3) $M_{1} \circ M_{2} \leq M_{1} \times M_{2}$, (4) $M_{1} \omega M_{2} \leq M_{1} \times M_{2}$.

Proof (1) Define $\eta: Q_{1} \times Q_{2} \rightarrow Q_{1} \times Q_{2}$ as an identity mapping of $Q_{1} \times Q_{2}$. Obviously the conclusion is true. 
(2) Define $\eta: Q_{1} \times Q_{2} \rightarrow Q_{1} \times Q_{2}$ as an identity mapping of $Q_{1} \times Q_{2}$. Obviouly $\eta$ is a partial function. Define $\quad \xi: X_{2} \rightarrow X_{1}^{Q_{2}} \times X_{2} \quad, \quad \xi(a)=(f, a), \forall a \in X_{2} \quad$,among $\quad$ which $f: Q_{2} \rightarrow X_{1}, f\left(p_{2}\right)=a=\omega\left(p_{2}, a\right), \forall p_{2} \in Q_{2}, \quad \xi$ as a function, and $\mu_{M_{1} \omega M_{2}}\left(\eta\left(q_{1}, q_{2}\right), a, \eta\left(p_{1}, p_{2}\right)\right)=\mu_{M_{1} \omega M_{2}}\left(\left(q_{1}, q_{2}\right), a,\left(p_{1}, p_{2}\right)\right)$ $=\mu_{M_{1}}\left(p_{1}, \omega\left(p_{2}, a\right), q_{1}\right) \wedge \mu_{M_{2}}\left(p_{2}, a, q_{2}\right)=\mu_{M_{1}}\left(p_{1}, f\left(p_{2}\right), q_{1}\right) \wedge \mu_{M_{2}}\left(p_{2}, a, q_{2}\right)$ $=\mu_{M_{1} \omega M_{2}}\left(\left(q_{1}, q_{2}\right),(f, a),\left(p_{1}, p_{2}\right)\right)=\mu_{M_{1} \omega M_{2}}\left(\left(q_{1}, q_{2}\right), \xi(a),\left(p_{1}, p_{2}\right)\right)$, for $M_{1} \omega M_{2} \leq M_{1} \circ M_{2}$.

(3)Define $\xi: X_{1}^{Q_{2}} \times X_{2} \rightarrow X_{1} \times X_{2}$ as $\xi(f, a)=\left(f\left(p_{2}\right), a\right)$, among which $f: Q_{2} \rightarrow X_{1}, f\left(q_{2}\right)=a_{1}, \forall a \in X_{2}, p_{2} \in Q_{2}$, and define $\eta$ as an identity mapping of $Q_{1} \times Q_{2}$, easy to prove $M_{1} \circ M_{2} \leq M_{1} \times M_{2}$.

(4) Based on(2)and(3), $M_{1} \omega M_{2} \leq M_{1} \times M_{2}$.

Theorem 4.2 Suppose $M_{i}=\left(Q_{i}, X_{i}, \mu_{i}\right)$ is a lattice-valued finite state machine, $i=1,2,3$.If $M_{1} \leq M_{2}$, then(1) $M_{1} \times M_{3} \leq M_{2} \times M_{3}, M_{3} \times M_{1} \leq M_{3} \times M_{2}$, and if $X_{1}=X_{2}=X_{3}=X$, then $M_{1} \wedge M_{3} \leq M_{2} \wedge M_{3}, \quad M_{3} \wedge M_{1} \leq M_{3} \wedge M_{2}$,(2)If among any $\omega_{1}: Q_{3} \times X_{3} \rightarrow X_{1}$, there exists $\omega_{2}: Q_{3} \times X_{3} \rightarrow X_{2}$, making $M_{1} \omega_{1} M_{3} \leq M_{2} \omega_{2} M_{3}$. If $(\eta, \xi)$ is a covering of $M_{2} \rightarrow M_{1}$, and $\xi$ is a surjection,then among any $\omega_{1}: Q_{1} \times X_{1} \rightarrow X_{3} \quad$, existing $\omega_{2}: Q_{2} \times X_{2} \rightarrow X_{3} \quad$, making $M_{3} \omega_{1} M_{1} \leq M_{3} \omega_{2} M_{2}$, (3) $M_{1} \circ M_{3} \leq M_{2} \circ M_{3}, M_{3} \circ M_{1} \leq M_{3} \circ M_{2}$.

\section{Acknowledgements}

Science and technology research project of Jiangxi Provincial Education Department (GJJ161223).

\section{References}

[1] Zadeh L A. Fuzzy sets [J].Information and Controls, 1965, 8(3):338-353.

[2] Mo Zhiwen,Pen Jiayin.An algorithm of test for fuzzy codes[J].Appl Math $\mathrm{J}$ Chinese Univser B,2001,16(1):88-94.

[3] Malik D S,Mordeson J N,Sen M K.Products of fuzzy finite state machines[J].Fuzzy Sets and Systems, 1997,92(2):95-102.

[4] DasP.On some properties of fuzzy semiautomata over a finite group [J].Inform Science, 1997, 101(1):71-84.

[5] Li Y M,.Lattice-valued automata and their languages[J].Journal of Shanxi Normal University(Natural Science Edition),2,2003,31(4):1-6.

[6] Liu J,Mo Z W.. Products of lattice-valued finite automata [J]. Applied Mathematics A Journal of Chinese Universities, 2209, 24 (1):121-126.

[7] Lei H X, Pan C. Lattice-valued finite automata and its properties [J]. Journal of Neijiang Teachers College (Natural Science Edition), 2006, 21(4):9-12.

[8] Lei H X. Homomorphism and quotient automata of lattice-valued finite states automata [J].Computer Engineering andApplications, 2010, 46(27):52-53. 